\title{
Screening for Alzheimer's Disease: Cognitive Impairment in Self-Referred and Memory Clinic-Referred Patients
}

\author{
Bjørn-Eivind Kirsebom ${ }^{\mathrm{a}, \mathrm{b}, *}$, Ragna Espenes ${ }^{\mathrm{a}, \mathrm{b}}$, Knut Waterloo $^{\mathrm{a}, \mathrm{b}}$, Erik Hessen $^{\mathrm{h}, \mathrm{i}}$, \\ Stein Harald Johnsen ${ }^{\mathrm{a}, \mathrm{c}}$, Geir Bråthen ${ }^{\mathrm{d}, \mathrm{e}}$, Dag Aarsland ${ }^{\mathrm{f}, \mathrm{g}, \mathrm{i}}$ and Tormod Fladby, ${ }^{\mathrm{i}, \mathrm{j}}$ \\ ${ }^{a}$ Department of Neurology, University Hospital of North Norway, Troms $\phi$, Norway \\ ${ }^{\mathrm{b}}$ Department of Psychology, Faculty of Health Sciences, UiT The Arctic University of Norway, Troms $\phi$, Norway \\ ${ }^{\mathrm{c}}$ Department of Clinical Medicine, Brain and Circulation Research Group, UiT The Arctic University \\ of Norway, Troms $\phi$, Norway \\ ${ }^{\mathrm{d}}$ Department of Neuromedicine and Movement Science, Faculty of Medicine and Health Sciences, \\ Norwegian University of Science and Technology, Trondheim, Norway \\ ${ }^{\mathrm{e}}$ Department of Neurology and Clinical Neurophysiology, University Hospital of Trondheim, Trondheim, Norway \\ ${ }^{\mathrm{f}}$ Centre for Age-Related Medicine, Stavanger University Hospital, Stavanger, Norway \\ ${ }^{\mathrm{g}}$ Department of Old Age Psychiatry, Institute of Psychiatry, Psychology and Neuroscience, \\ King's College London, London, UK \\ ${ }^{\mathrm{h}}$ Department of Psychology, University of Oslo, Oslo, Norway \\ ${ }^{i}$ Department of Neurology, Akershus University Hospital, Lørenskog, Norway \\ ${ }^{\mathrm{j}}$ Institute of Clinical Medicine, Campus Ahus, University of Oslo, Oslo, Norway
}

Handling Associate Editor: Frank Jessen

Accepted 21 August 2017

\begin{abstract}
.
Background: Cognitive assessment is essential in tracking disease progression in AD. Presently, cohorts including preclinical at-risk participants are recruited by different means, which may bias cognitive and clinical features. We compared recruitment strategies to levels of cognitive functioning.

Objective: We investigate recruitment source biases in self-referred and memory clinic-referred patient cohorts to reveal potential differences in cognitive performance and demographics among at-risk participants.

Methods: We included 431 participants $40-80$ years old. Participants were classified as controls $(n=132)$ or symptom group $(n=299)$. The symptom group comprised of subjective cognitive decline (SCD, $n=163)$ and mild cognitive impairment (MCI, $n=136)$. We compared cognitive performance and demographics in memory clinic-referrals $(n=86)$ to self-referred participants responding to advertisements and news bulletins $(n=179)$. Participants recruited by other means were excluded from analysis $(n=34)$.

Results: At symptom group level, we found significant reductions in cognitive performance in memory clinic-referrals compared to self-referrals. However, here reductions were only found within the MCI group. We found no differences in cognitive performance due to recruitment within the SCD group. The MCI group was significantly impaired compared to controls on all measures. Significant reductions in learning, and executive functions were also found for the SCD group.
\end{abstract}

\footnotetext{
${ }^{*}$ Correspondence to: Bjørn-Eivind Kirsebom, Department of Neurology, University Hospital of North Norway, UNN, 9037
}

$\overline{\text { Tromsø, Norway. Tel.: +47 93054791; E-mail: bjorneivind.kirse }}$ bom@gmail.com. 
Conclusion: Regardless of recruitment method, both the SCD and MCI groups showed reductions in cognitive performance compared to controls. We found differences in cognitive impairment for memory clinic-referrals compared to self-referrals only within the MCI group, SCD-cases being equally affected irrespective of referral type.

Keywords: Alzheimer's disease, cognitive dysfunction, mild cognitive impairment, patient recruitment, research subject recruitment, sampling studies, subjective cognitive decline

\section{INTRODUCTION}

Mild cognitive impairment (MCI) is associated with an increased risk for Alzheimer's disease (AD). MCI due to AD constitutes a transitory phase between normal cognitive function and dementia [1]. Research efforts have aimed to define features and trajectories of $\mathrm{MCI}$ due to $\mathrm{AD}$ to predict conversion from $\mathrm{MCI}$ to $\mathrm{AD}$, and to distinguish $\mathrm{MCI}$ due to $\mathrm{AD}$ from other causes of MCI such as vascular disease, early frontotemporal dementia, or early stages of dementia with Lewy bodies [1,2]. Converging evidence from studies of at-risk cohorts and clinically normal older individuals now indicate that the pathophysiological underpinnings of AD may begin 10 to 15 years before the emergence of clinical symptoms [3]. Consequently, this has led to the proposal that $\mathrm{AD}$ has a preclinical phase wherein brain-compensatory mechanisms make up for early pathological changes [4]. Identifying individuals at risk for AD in the preclinical phase is a key objective [5, 6]. Future effective treatments at this level could serve to preserve or delay onset of objective cognitive decline $[4,7,8]$.

A proposed target population for preclinical AD is patients with subjective experience of cognitive deficits, hypothesizing that subjective cognitive decline (SCD), i.e., with normal performance on standardized cognitive tests, may imply risk for conversion to $\mathrm{MCI}$ and ultimately $\mathrm{AD}$ dementia [6]. SCD manifests before the onset of clinical identifiable impairment, such as objective cognitive decline and could potentially serve as a target population for early intervention trials. Indeed, several longitudinal studies have shown that SCD carries a small, but detectable risk of conversion to MCI [9-11]. A recent systematic review of subjective cognitive complaints (SCC) risk to $\mathrm{AD} / \mathrm{MCI}$ progression reported that 16 out of 17 studies showed a 1.5- to 3-fold higher risk of progression in patients 59 years or older [12]. However, it should also be noted that the overwhelming majority of studies did not show progression from SCC to objective cognitive decline (MCI or Dementia) when assessed at follow-up. Bassett and Folstein [13] have shown that up to $43 \%$ of those aged between
65 and 74 years report subjective memory problems, while dementia prevalence in this age range is low. Thus, in many cases, the experience of cognitive decline is either benign, or caused by other conditions or disorders than $\mathrm{AD}$. Consequently, there is a need to identify the characteristics of SCD due to $\mathrm{AD}$ and other disorders, in order to identify preclinical at-risk populations eligible for early intervention and intervention trials [6].

In order to improve on research criteria for SCD, The Subjective Cognitive Decline working group (SCD-I) [6] have proposed a conceptual framework for research on SCD as a preclinical risk factor for AD. Among several issues, they underline that differences in research setting, design, and participant selection may influence the composition of clinical characteristics within at-risk cohorts. Cohorts including at-risk participants are recruited by different means, which lead to inclusion of cohorts with different clinical and demographic characteristics. It has been demonstrated that MCI participants recruited through memory clinics harbor more AD-type pathology [14], show a higher prevalence of APOE $\varepsilon 4$ alleles [15], are cognitively more impaired [15], and have higher risk of progression to dementia $[16,17]$ than participants recruited through community or population based samples. Moreover, volunteer sample controls have shown to be better educated and perform better on cognitive tests than population-based samples [15]. However, few studies have investigated the effects of recruitment bias in patients with SCD [18]. Chen et al. [19] recently demonstrated that persons with normal cognitive scores at baseline, showed an annual conversion rate to $\mathrm{MCI}$ of $30 \%$ in a memory clinic sample compared to $5 \%$ in a community based sample. They attribute this finding to level of concern leading to medical help seeking within the memory clinic sample. Similarly, Perrotin et al. [20] recently published findings demonstrating reduced gray matter volumes and increased depressive symptomatology in SCD cases from a memory clinic sample compared to community-sample. These studies did not demonstrate any differences in cognitive performance at baseline due to recruitment bias. In contrast, 
Abdelnour et al. [21] recently showed reduced cognitive performance in SCD cases from a memory-unit compared to cases recruited from an open house initiative offering free examinations to the community. These findings demonstrate a need to explore potential differences in clinical characteristics within and between preclinical cohorts employing different recruitment strategies. SCD is a particularly vulnerable clinical group, as many cases ultimately are not related to AD pathology [12, 13, 22].

This study compares cognitive performance and demographic characteristics between at-risk participants recruited through memory clinics and participants self-referred by voluntary response to news-bulletins and advertisement. We hypothesize that participants recruited through referrals by a general practitioner (GP) to memory outpatient clinics are more cognitively impaired than participants that are self-referred by voluntary response to newsbulletins or advertisement. We further explore these cohorts by comparing the cognitive symptom groups $\mathrm{MCI}$ and SCD to a control group.

\section{METHODS}

This study was a part of "Dementia Disease Initiation" (DDI), a co-operation between all Norwegian health regions and university hospitals. Between January 2013 and January 2017, we recruited participants with self-reported cognitive reduction and healthy controls. For further description of the DDI cohort and methods, see Fladby et al. [23]. All participants were examined following a standard protocol. Participants were recruited from two main sources: 1) self-referred, following advertisements in media, newspapers, or news bulletins, or 2) GP referrals to local memory clinics. Additionally, cognitively healthy controls were included from spouses of patients with dementia/cognitive disorder, and from patients who completed lumbar puncture for orthopedic surgery. Participants were classified as controls, SCD or MCI according to criteria based on a comprehensive assessment program (see below) [6, 24]. The controls were further classified as having either normal or abnormal cognitive screening, and with or without first-degree relative with dementia. We included individuals with a native language of Norwegian, Swedish, or Danish. In order to capture individuals in the preclinical, as well as predementia phases of $\mathrm{AD}$, we included participants between 40 and 80 years of age. Exclusion criteria were brain trauma or disorder, including clinical stroke, dementia, severe psychiatric disorder, severe somatic disease that might influence the cognitive functions, or intellectual disability or other developmental disorders.

A case report form developed for DDI [23] included assessment protocol for SCD (see below), medical history from participant and informant, physical and neurological examinations, as well as the 15-item Geriatric Depression Score (GDS) [25]. Educational levels were classified in the following categories [26]: $0=$ Primary school (7-8 y), $1=$ High School $(9-11 \mathrm{y}), 2=$ College $(12 \mathrm{y}), 3=$ Bachelor degree $(13-15 \mathrm{y}), 4=$ Master or equivalent $=16-$ $17 \mathrm{y}, 5=$ Higher university degree/PhD (18-20y). The cognitive examination included the Mini-Mental State Examination (MMSE-NR) [27], non-verbal cognitive screening (The clock drawing test) [28], verbal memory (CERAD word list) [29], visuoperceptual ability (VOSP silhouettes) [32], psychomotor speed and divided attention (Trail making A and B), and word fluency (COWAT) [30].

The regional medical research ethics committee approved the study. All participants gave their written informed consent before taking part in the study. All further study conduct was in line with the guidelines provided by the Helsinki declaration of 1964 (revised 2013) and the Norwegian Health and Research Act.

\section{Classification of SCD and MCI}

The DDI case report form [23] includes a comprehensive account of participants' experience of subjective cognitive decline modelled on the suggested framework by the working group of SCD-I. It includes the nature of cognitive decline (cognitive domain, onset), concerns and worries, including feeling worse compared to age matched peers, and informant confirmation of decline (when available). Participants were classified as SCD according to the SCD-I framework, which requires normal objective cognitive performance in combination with subjectively experienced decline in any cognitive domain [6]. MCI was classified according to the NIA-AA criteria which require presence of subjective cognitive decline or impairment combined with lower performance than expected in one or more cognitive domains, yet preserved independence in functional ability and not fulfilling the criteria of dementia $[24,31]$. Performance was classified as normal or abnormal according to published norms (adjusted for age, sex, and educational effects) for the different 
tests [27-30, 32-34]. Due to overlapping and mutually exclusive criteria, the cut-off values for SCD versus MCI (defined as normal or abnormal cognition) were $\leq 1.5$ standard deviation below normative mean on either CERAD word list (delayed recall), VOSP silhouettes, TMT-B or COWAT, or having MMSE score equal to or below 27. Cognitive functioning was also assessed by the Clinical Dementia Rating scale (CDR) [35]. Participants with dementia were excluded if CDR > 0.5 [36].

\section{Participants}

Of 577 participants considered, 87 were excluded because they withdrew before finishing the assessment program or did not fulfil the inclusion criteria. Of the 490 participants included, 463 were classified according to disease stage at the time of analysis (Fig. 1). Participants were classified as normal control $(n=132$, mean age $=60.4, \mathrm{SD}=9.3)$ or cognitive symptom group $(n=299$, mean age $=63.7$, $\mathrm{SD}=9.4)$, the latter comprising of symptom subgroups MCI $(n=136$, mean age $=65.4, \mathrm{SD}=9.8)$ and $\operatorname{SCD}(n=163$, mean age $=62.3, \mathrm{SD}=8.9)$. Participants who were recruited as normal controls, but had abnormal cognitive screening were excluded from analysis $(n=32)$. Following advertisements in media, we recruited 179 self-referred participants (mean age $=64.4, \mathrm{SD}=9.7$ ), whereas 86 participants (mean age $=61.5, \mathrm{SD}=9.1$ ) were recruited among referrals to local memory clinics. Participants recruited by other means or when recruitment source was not available were excluded from analysis $(n=34)$ [23].

\section{Statistical analysis}

For variables with assumed normal distribution (age at inclusion, CERAD word list learning \& recall, T-score, VOSP silhouettes T-score, TMT A \& B Tscore, and COWAT T-score), we compared means for the different groups with one-way ANOVA (analysis of variance) and calculated effect sizes using eta squared $\left(\eta \mathrm{p}^{2}\right)$. We assessed normality by visual inspection of frequency distributions, Q-Q-plots, and box-plots. Assessing variables with Levene's test, equal variance was assumed for all variables except CERAD word list delayed recall and VOSP silhouettes T-score. Continuous variables with non-normal distribution (MMSE, Clock drawing test) were compared with Mann-Whitney U tests. Education level, being an ordinal variable, was also tested with Mann-Whitney U. We used the Mann-Whitney U

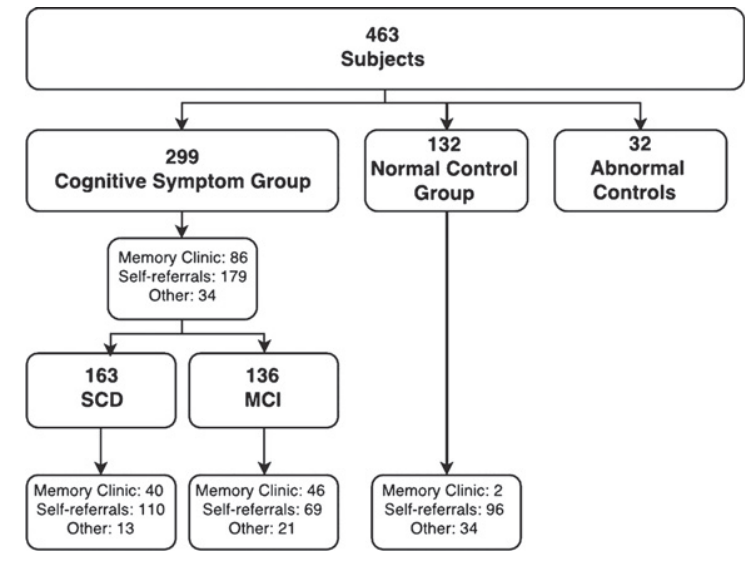

Fig. 1. A total of 463 participants were classified according to disease stage at the time of analysis, whereof 32 recruited as controls showed abnormal cognitive performance and were excluded from analysis. Participants were classified as belonging to a normal control group or cognitive symptom group (SCD and $\mathrm{MCI}$ ), and their characteristics analyzed depending on recruitment source.

Z-statistic to calculate effect size $\left(R=\frac{Z}{\sqrt{N}}\right)$ [37]. We compared the binary variable "sex", with Pearson's Chi square test. All analyses were performed in the Statistical Package for Social Sciences (SPSS) version 24 .

\section{RESULTS}

\section{Cognitive performance compared to recruitment strategy}

All data pertaining to comparisons of cognitive performance to recruitment strategies, including demographic characteristics, are shown in Table 1.

No differences in gender distributions were shown between recruitment sources. However, at symptom group level, including both SCD and MCI participants, memory clinic-referrals were shown to be both younger $\left(p<0.05, \eta \mathrm{p}^{2}=0.020\right)$ and less educated $(p<0.01, r=0.160)$ than self-referrals. When measuring the SCD and MCI groups separately, this recruitment bias was only shown for memory clinic-referred MCI participants [age $\left(p<0.001, \eta \mathrm{p}^{2}=0.089\right)$ and education level $(p<0.05$, $r=0.201)]$. We found no differences in demographic characteristics between recruitment strategies within the SCD group.

At symptom group level, including both SCD and MCI participants, memory clinic-referrals performed significantly worse than self-referrals on MMSE $(p<0.05, r=0.138)$, Clock drawing test $(p<0.01$, 
Table 1

Demographic characteristics and cognitive test results comparisons between recruitment strategies within the cognitive symptom group (MCI and SCD) and symptom subgroups mild cognitive impairment (MCI) and subjective cognitive decline (SCD)

\begin{tabular}{|c|c|c|c|c|c|c|}
\hline & \multicolumn{2}{|c|}{$\begin{array}{c}\text { Cognitive symptom Group } \\
\text { (MCI \& SCD) }\end{array}$} & \multicolumn{2}{|c|}{ MCI } & \multicolumn{2}{|c|}{ SCD } \\
\hline & Self-referral & $\begin{array}{c}\text { Memory } \\
\text { clinic referral }\end{array}$ & Self-referral & $\begin{array}{c}\text { Memory } \\
\text { clinic referral }\end{array}$ & Self-referral & $\begin{array}{c}\text { Memory } \\
\text { clinic referral }\end{array}$ \\
\hline Age at inclusion & $n=179$ & $n=86$ & $n=69$ & $n=46$ & $n=110$ & $n=40$ \\
\hline Mean $(S D)$ & $64.4(9.7)$ & $\begin{array}{c}61.5(9.1) \\
\boldsymbol{p}<\mathbf{0 . 0 5}^{a} \\
\eta \mathbf{p}^{2}=\mathbf{0 . 0 2 0}\end{array}$ & $67.4(9.3)$ & $\begin{array}{c}61.3(10.3) \\
\boldsymbol{p}<\mathbf{0 . 0 0 1}^{a} \\
\eta \mathbf{p}^{2}=\mathbf{0 . 0 8 9}\end{array}$ & $62.5(9.6)$ & $\begin{array}{c}61.7(7.7) \\
p=\text { n.s. }^{a}\end{array}$ \\
\hline Female/Total & $96 / 179$ & $45 / 86$ & $33 / 69$ & $21 / 46$ & $63 / 110$ & $24 / 40$ \\
\hline Percentage female & $53.6 \%$ & $\begin{array}{c}52.3 \% \\
p=\text { n.s. }^{c}\end{array}$ & $47.8 \%$ & $\begin{array}{c}45.7 \% \\
p=\text { n.s. }^{c}\end{array}$ & $57.3 \%$ & $\begin{array}{c}60.0 \% \\
p=\text { n.s. }{ }^{c}\end{array}$ \\
\hline Education level & $n=178$ & $n=86$ & $n=68$ & $n=46$ & $n=110$ & $n=40$ \\
\hline Median $(I Q R)$ & $3.0(2.0)$ & $\begin{array}{l}2.5(2.0) \\
\boldsymbol{p}<\mathbf{0 . 0 1}^{b} \\
\boldsymbol{r}=\mathbf{0 . 1 6 0}\end{array}$ & $3.0(2.0)$ & $\begin{array}{l}2.5(2.0) \\
\boldsymbol{p}<\mathbf{0 . 0 5}^{b} \\
\boldsymbol{r}=\mathbf{0 . 2 0 1}\end{array}$ & $3.0(2.0)$ & $\begin{array}{c}2.5(2.75) \\
p=\text { n.s. }^{b}\end{array}$ \\
\hline MMSE & $n=178$ & $n=85$ & $n=69$ & $n=45$ & $n=109$ & $n=40$ \\
\hline Mean $(S D)$ & $28.7(1.5)$ & $\begin{array}{l}28.1(1.9) \\
\boldsymbol{p}<\mathbf{0 . 0 5}^{b} \\
\boldsymbol{r}=\mathbf{0 . 1 3 8}\end{array}$ & $28.0(1.8)$ & $\begin{array}{c}27.3(2.2) \\
p=\text { n.s. }{ }^{b}\end{array}$ & $29.2(1.1)$ & $\begin{array}{c}29.1(1.0) \\
p=\text { n.s. }\end{array}$ \\
\hline Clock Drawing Test & $n=178$ & $n=84$ & $n=69$ & $n=45$ & $n=109$ & $n=39$ \\
\hline Mean $(S D)$ & $4.9(0.4)$ & $\begin{array}{l}4.7(0.6) \\
\boldsymbol{p}<\mathbf{0 . 0 1}^{b} \\
\boldsymbol{r}=\mathbf{0 . 1 8 8}\end{array}$ & $4.8(0.5)$ & $\begin{array}{l}4.6(0.8) \\
\boldsymbol{p}<\mathbf{0 . 0 5}^{b} \\
\boldsymbol{r}=\mathbf{0 . 1 8 6}\end{array}$ & $4.9(0.3)$ & $\begin{array}{c}4.8(0.4) \\
p=\mathrm{n} . \mathrm{s}^{b}\end{array}$ \\
\hline CERAD word list Learning $\mathrm{T}$ score & $n=177$ & $n=84$ & $n=68$ & $n=45$ & $n=109$ & $n=39$ \\
\hline $\operatorname{Mean}(S D)$ & $49.7(12.0)$ & $\begin{array}{c}43.5(14.5) \\
\boldsymbol{p}<\mathbf{0 . 0 0 1}^{a} \\
\eta \mathbf{p}^{2}=\mathbf{0 . 0 4 9}\end{array}$ & $43.1(12.7)$ & $\begin{array}{c}36.0(12.4) \\
\boldsymbol{p}<\mathbf{0 . 0 1}^{a} \\
\eta \mathbf{p}^{2}=\mathbf{0 . 0 7 3}\end{array}$ & $53.8(9.6)$ & $\begin{array}{c}52.1(11.7) \\
p=\text { n.s. }^{a}\end{array}$ \\
\hline CERAD word list Recall T-score & $n=176$ & $n=81$ & $n=68$ & $n=41$ & $n=108$ & $n=40$ \\
\hline Mean $(S D)$ & $47.8(13.5)$ & $\begin{array}{c}42.7(15.1) \\
\boldsymbol{p}<\mathbf{0 . 0 1}^{a} \\
\eta \mathbf{p}^{2}=\mathbf{0 . 0 3 0}\end{array}$ & $39.5(14.2)$ & $\begin{array}{c}34.4(13.5) \\
p=\text { n.s. }^{a}\end{array}$ & $53.3(9.9)$ & $\begin{array}{c}51.2(11.5) \\
p=\text { n.s. }^{a}\end{array}$ \\
\hline VOSP Silhouettes T-score & $n=169$ & $n=70$ & $n=65$ & $n=41$ & $n=104$ & $n=29$ \\
\hline Mean $(S D)$ & $49.6(11.2)$ & $\begin{array}{l}46.5(11.6) \\
p=\mathrm{n}^{a} \mathrm{~s}^{a}\end{array}$ & $44.4(11.0)$ & $\begin{array}{l}42.4(11.2) \\
p=\text { n.s. }^{a}\end{array}$ & $52.9(10.0)$ & $\begin{array}{l}52.4(9.3) \\
p=\text { n.s. }^{a}\end{array}$ \\
\hline Trail Making Test A & $n=177$ & $n=85$ & $n=68$ & $n=45$ & $n=109$ & $n=40$ \\
\hline T-score & $45.1(10.3)$ & $45.4(10.3)$ & $40.4(10.2)$ & $41.4(10.5)$ & $48.0(9.4)$ & $50.0(8.2)$ \\
\hline Mean $(S D)$ & & $p=$ n.s. $^{a}$ & & $p=$ n.s. $^{a}$ & & $p=$ n.s. $^{a}$ \\
\hline Trail Making Test B & $n=177$ & $n=85$ & $n=68$ & $n=45$ & $n=109$ & $n=40$ \\
\hline T-score & $45.9(11.1)$ & $42.5(12.3)$ & $40.3(11.8)$ & $37.0(13.0)$ & $49.5(9.0)$ & $48.7(7.7)$ \\
\hline Mean $(S D)$ & & $\begin{array}{c}p<\mathbf{0 . 0 5}^{a} \\
\eta \mathbf{p}^{2}=\mathbf{0 . 0 1 9}\end{array}$ & & $p=$ n.s. ${ }^{a}$ & & $p=$ n.s. $^{a}$ \\
\hline $\begin{array}{l}\text { Controlled Oral Word } \\
\text { Association Test } \\
\text { (COWAT) T-Score }\end{array}$ & $n=176$ & $n=84$ & $n=68$ & $n=44$ & $n=108$ & $n=40$ \\
\hline Mean (SD) & $49.6(10.1)$ & $\begin{array}{c}47.5(10.7) \\
p=\text { n.s. }^{a}\end{array}$ & $46.6(10.0)$ & $\begin{array}{l}44.2(10.6) \\
p=\text { n.s. }^{a}\end{array}$ & $51.5(9.7)$ & $\begin{array}{l}51.0(9.7) \\
p=\text { n.s. }^{a}\end{array}$ \\
\hline
\end{tabular}

The continuous variables (Age at inclusion, CERAD word list learning and recall T-score, VOSP silhouettes T-score, TMT-A and TMT-B $T$-score and COWAT T-score) are summarized by mean (standard deviation, SD). The ordinal variable educational level is described by median (interquartile range). Variables of assumed normal distribution are compared with one-way ANOVA with predefined contrasts and effect sizes $\left(\eta \mathrm{p}^{2}\right)$ are provided for significant results (a). Variables of non-normal distribution (MMSE and Clock drawing test) and the ordinal variable (education level) are compared with Mann-Whitney $\mathrm{U}$ tests and effect sizes ( $\mathrm{r}$ ) are provided for significant results (b). The binary variable sex is described with observed numbers and percentages and compared with Pearson's Chi square tests (c). Significant $p$-values and effect sizes are shown in Bold.

$r=0.188)$, CERAD word list learning $(p<0.001$, $\left.\eta \mathrm{p}^{2}=0.049\right)$, CERAD word list recall $(p<0.01$, $\left.\eta \mathrm{p}^{2}=0.030\right)$, and trail making test $\mathrm{B}(p<0.05$, $\left.\eta \mathrm{p}^{2}=0.019\right)$. However, within the MCI group, this performance deficit was only shown for the clock drawing test $(p<0.05, r=0.186)$ and CERAD word list learning $\left(p<0.01, \eta \mathrm{p}^{2}=0.073\right)$. Within the SCD group we found no significant differences in cognitive performance between self-referrals and memory clinic referrals. 
Table 2

Demographic characteristics and cognitive test result comparisons between control group and cognitive symptom group (SCD and $\mathrm{MCI}$ ), as well as subgroups with mild cognitive impairment (MCI) and subjective cognitive decline (SCD)

\begin{tabular}{|c|c|c|c|c|}
\hline & Control Group & $\begin{array}{c}\text { Cognitive } \\
\text { symptom Group } \\
\text { (MCI \& SCD) }\end{array}$ & MCI & $\mathrm{SCD}$ \\
\hline Age at inclusion & $n=132$ & $n=299$ & $n=136$ & $n=163$ \\
\hline \multirow[t]{2}{*}{ Mean $(S D)$} & $60.4(9.3)$ & $63.7(9.4)$ & $65.4(9.8)$ & $62.3(8.9)$ \\
\hline & & $\begin{array}{c}p<\mathbf{0 . 0 0 1}^{a} \\
\eta \mathbf{p}^{2}=\mathbf{0 . 0 2 6}\end{array}$ & $\begin{array}{c}p<\mathbf{0 . 0 0 1}^{a} \\
\eta \mathbf{p}^{2}=0.064\end{array}$ & $p=\mathrm{n}_{\mathrm{s}} .^{a}$ \\
\hline Female/Total & $75 / 132$ & $155 / 299$ & $63 / 136$ & $92 / 163$ \\
\hline \multirow[t]{2}{*}{ Percentage female } & $56.8 \%$ & $51.8 \%$ & $46.3 \%$ & $56.4 \%$ \\
\hline & & $p=\mathrm{n} .{ }^{c}{ }^{c}$ & $p=$ n.s. $^{c}$ & $p=\mathrm{n} . \mathrm{s}^{c}{ }^{c}$ \\
\hline Education level & $n=131$ & $n=298$ & $n=135$ & $n=163$ \\
\hline \multirow[t]{2}{*}{ Median (IQR) } & $3.0(2.0)$ & $3.0(2.0)$ & $3.0(3.0)$ & $3.0(3.0)$ \\
\hline & & $p=\mathrm{n} . \mathrm{s} .{ }^{b}$ & $p=\mathrm{n} . \mathrm{s} .{ }^{b}$ & $p=\mathrm{n} . \mathrm{s} .{ }^{b}$ \\
\hline MMSE & $n=131$ & $n=296$ & $n=134$ & $n=162$ \\
\hline \multirow[t]{3}{*}{ Mean (SD) } & $29.4(0.9)$ & $28.4(1.7)$ & $27.6(2.0)$ & $29.1(1.1)$ \\
\hline & & $\boldsymbol{p}<\mathbf{0 . 0 0 1}^{b}$ & $\boldsymbol{p}<\mathbf{0 . 0 0 1}^{b}$ & $\boldsymbol{p}<\mathbf{0 . 0 5}^{b}$ \\
\hline & & $r=0.278$ & $r=0.514$ & $r=0.126$ \\
\hline Clock Drawing Test & $n=130$ & $n=293$ & $n=134$ & $n=159$ \\
\hline \multirow[t]{3}{*}{ Mean (SD) } & $4.9(0.3)$ & $4.8(0.5)$ & $4.7(0.6)$ & $4.9(0.3)$ \\
\hline & & $\boldsymbol{p}<\mathbf{0 . 0 1}^{b}$ & $p<0.001^{b}$ & $p=\mathrm{n}^{\mathrm{s}} .^{b}$ \\
\hline & & $\mathbf{r}=\mathbf{0 . 1 3 0}$ & $r=0.250$ & \\
\hline CERAD word list Learning $\mathrm{T}$ score & $n=130$ & $n=293$ & $n=133$ & $n=160$ \\
\hline \multirow[t]{2}{*}{ Mean $(S D)$} & $56.1(9.5)$ & $47.8(13.0)$ & $40.9(12.8)$ & $53.5(10.2)$ \\
\hline & & $\begin{array}{c}p<\mathbf{0 . 0 0 1}^{a} \\
\eta \mathbf{p}^{2}=\mathbf{0 . 0 9 2}\end{array}$ & $\begin{array}{c}p<\mathbf{0 . 0 0 1}^{a} \\
\eta \mathbf{p}^{2}=\mathbf{0 . 3 1 3}\end{array}$ & $\begin{array}{c}p<\mathbf{0 . 0 5}^{a} \\
\eta \mathbf{p}^{2}=\mathbf{0 . 0 1 6}\end{array}$ \\
\hline CERAD word list Recall T-score & $n=130$ & $n=289$ & $n=130$ & $n=160$ \\
\hline \multirow[t]{3}{*}{ Mean $(S D)$} & $55.2(11.2)$ & $46.1(14.5)$ & $37.5(14.4)$ & $53.0(10.3)$ \\
\hline & & $p<\mathbf{0 . 0 0 1}^{a}$ & $p<\mathbf{0 . 0 0 1}^{a}$ & $p=$ n.s. ${ }^{a}$ \\
\hline & & $\eta \mathbf{p}^{2}=\mathbf{0 . 0 8 7}$ & $\eta \mathbf{p}^{2}=\mathbf{0 . 3 2 0}$ & \\
\hline VOSP Silhouettes T-score & $n=120$ & $n=268$ & $n=126$ & $n=142$ \\
\hline \multirow[t]{2}{*}{ Mean $(S D)$} & $52.7(8.9)$ & $48.2(11.2)$ & $43.6(10.9)$ & $52.3(9.8)$ \\
\hline & & $\begin{array}{c}p<0.001^{a} \\
n \mathbf{n}^{2}=0.038\end{array}$ & $\begin{array}{c}p<0.001^{a} \\
n \mathbf{p}^{2}=0.175\end{array}$ & $p=\mathrm{n} \cdot \mathrm{s}^{a}$ \\
\hline Trail Making Test A & $n=128$ & $\begin{array}{c}n \mathrm{p}=\mathbf{0 . 0 0 0} \\
n=293\end{array}$ & $\begin{array}{c}n \mathbf{p}=\mathbf{n} \\
n=132\end{array}$ & $n=161$ \\
\hline T-score & $49.2(9.6)$ & $45.0(10.2)$ & $40.7(9.9)$ & $48.6(8.9)$ \\
\hline Mean (SD) & & $\begin{array}{c}p<\mathbf{0 . 0 0 1}^{a} \\
\eta \mathbf{p}^{2}=\mathbf{0 . 0 3 5}\end{array}$ & $\begin{array}{c}p<\mathbf{0 . 0 0 1}^{a} \\
\eta \mathbf{p}^{2}=\mathbf{0 . 1 5 7}\end{array}$ & $p=$ n.s. $^{a}$ \\
\hline Trail Making Test B & $n=127$ & $n=293$ & $n=132$ & $n=161$ \\
\hline T-score & $51.6(8.8)$ & $44.9(11.7)$ & $39.5(12.4)$ & $49.4(9.0)$ \\
\hline \multirow[t]{2}{*}{ Mean $(S D)$} & & $p<\mathbf{0 . 0 0 1}^{a}$ & $p<0.001^{a}$ & $p<0.05^{a}$ \\
\hline & & $\eta \mathbf{p}^{2}=0.074$ & $\eta \mathbf{p}^{2}=\mathbf{0 . 2 4 3}$ & $\eta \mathbf{p}^{2}=\mathbf{0 . 0 1 5}$ \\
\hline Controlled Oral Word & $n=128$ & $n=290$ & $n=131$ & $n=159$ \\
\hline Association Test & $50.5(7.6)$ & $48.8(10.4)$ & $45.5(10.1)$ & $51.6(9.8)$ \\
\hline (COWAT) T-Score & & $p=$ n.s. $^{a}$ & $p<0.001^{a}$ & $p=$ n.s. ${ }^{a}$ \\
\hline Mean $(S D)$ & & & $\eta \mathbf{p}^{2}=\mathbf{0 . 0 7 2}$ & \\
\hline
\end{tabular}

The continuous variables (Age at inclusion, CERAD word list learning and recall T-score, VOSP silhouettes T-score, TMT-A and TMT-B T-score and COWAT T-score) are summarized by mean (standard deviation, SD). The ordinal variable educational level is described by median (interquartile range). Variables of assumed normal distribution are compared with one-way ANOVA with predefined contrasts and effect sizes $\left(\eta \mathrm{p}^{2}\right)$ are provided for significant results (a). Variables of non-normal distribution (MMSE and Clock drawing test) and the ordinal variable (education level) are compared with Mann-Whitney U tests and effect sizes (r) are provided for significant results (b). The binary variable sex is described with observed numbers and percentages and compared with Pearson's Chi square tests (c). Significant $p$-values and effect sizes are shown in Bold.

\section{Cognitive symptom groups compared to control group}

All data pertaining to control and symptom group comparisons including demographic characteristics are shown in Table 2.
There were no differences in educational level or gender distributions between groups. However, the symptom group was significantly older than the control group $\left(p<0.001, \eta p^{2}=0.026\right)$. This difference was attenuated when comparing the MCI group to controls $\left(p<0.001, \eta \mathrm{p}^{2}=0.064\right)$, but not 
significant when comparing the SCD group to controls. Moreover, a further analysis of the control group characteristics showed that a majority was recruited through the spouses of participants responding to advertisements ( $n=96,72.7 \%$ ), whereas $25.8 \%$ $(n=34)$ were recruited by other means, and only $1.5 \%$ $(n=2)$ were recruited through the spouses of memory clinic-referrals.

At symptom group level, including both MCI and SCD participants, we found significantly reduced test performances compared to controls on all measures $(p<0.01)$, except the controlled oral word association test (COWAT). When comparing controls to MCI participants, significant reductions were found for all measures, including COWAT $(p<0.001$, see Table 2 for details). SCD participants performed significantly worse compared to controls on MMSE $(p<0.05, r=0.126)$, CERAD word list learning $\left(p<0.05, \eta \mathrm{p}^{2}=0.016\right)$, and trail making test $\mathrm{B}$ $\left(p<0.05, \eta \mathrm{p}^{2}=0.015\right)$.

\section{DISCUSSION}

In agreement with our hypothesis, memory clinicreferrals performed worse on cognitive tests than self-referred individuals. In addition, they were both younger and less educated compared to self-referrals. However, when comparing the MCI and SCD groups separately, only the MCI group showed these recruitment biases. We found no differences in cognitive performance or demographic characteristics between recruitment strategies within the SCD group. Further, we found that the SCD group performed worse on both cognitive screening (MMSE), and the cognitive subtests; word list learning (CERAD), and divided attention (TMT-B) compared to an age and education matched control group. In addition, the MCI group was shown to be older compared to controls, but did not differ in gender distributions or educational level.

In line with earlier reports, our findings show that including at-risk patients from memory clinics preferentially recruit individuals who are more cognitively impaired compared to self-referred individuals from the community. These findings generally support the notion that inclusion from memory clinics recruit individuals that are at higher risk of conversion to dementia $[16,17]$ or are farther along the disease trajectory than participants recruited through other means $[14,15]$. Moreover, the MCI participants recruited through memory clinics, while more cognitively impaired, were also younger and might represent an earlier onset, or more aggressive form of pathology than found in the older self-referred sample. Memory clinic samples have shown to harbor higher risk in terms of genetic risk factors [15], higher presence of AD-type pathology [38], or more aggressive forms of pathology [14]. However, the memory clinic-referred MCI cases in our sample had a lower educational level than their self-referred counterparts. Educational level is associated with cognitive reserve [39], thus lower cognitive performance in this group may be confounded with a lesser ability to compensate for brain pathology compared to the selfreferred group. Lastly, our control group comprised nearly $73 \%$ advertisement recruited individuals. It has been shown that controls recruited from convenience samples tend to be younger, better educated, and perform better on cognitive tests than controls recruited through population samples [15]. As such, our control group may not be an adequate comparison to memory clinic-referrals. However, although the cognitive symptom group was found to be older than controls, no difference was found in educational levels. Moreover, apart from the clock drawing test and MMSE, cognitive test scores were adjusted for effects of age and educational levels making between group comparisons possible.

We found no significant differences in either demographics or cognitive performance due to recruitment bias within the SCD group. This is perhaps not surprising given the fact that a core criterion for SCD is normal range of scores on neuropsychological examinations [6]. Any subtle differences between recruitment methods may be too small for detection within this group. Furthermore, recruitment did not bias other key demographics, leaving self- and memory clinic-referrals matched on these variables. To our knowledge, only one study to date have shown recruitment source to preferentially bias cognitive performance in SCD cases [21]. However, there are key differences in sample characteristics between studies. While both studies recruited participants from memory clinics, Abdelnour et al. [21] recruited participants from an open house initiative (OHI). This initiative offered free examinations to the community and did not specifically recruit participants to a memory study. In addition, the OHI SCD cases were more likely to be female and had higher educational levels compared to the memory unit sample. Conversely, regardless of recruitment source, participants within the DDI cohort were recruited specifically for a study on cognitive reduction. Moreover, we found no significant recruitment bias in demographics within the 
SCD group. Thus, regardless of recruitment source, the DDI SCD participants may be more similar within the DDI cohort, and thereby showing similar levels of cognitive performances. However, although notreaching the level of statistical significance, the data did show a trend towards both subtle lower performance and lower educational level in memory clinic-referred SCD cases compared to self-referrals. The lack of statistical significance for this result may be due to a small sample size (memory clinic-referred SCD cases $(n=40)$ ), and could have reached statistical significance given a larger sample. Moreover, we did find an overall significant difference in cognitive performance at symptom group level beyond what was shown by the MCI group alone. This suggests that although the differences are small, SCD cases recruited from memory clinics may represent a cognitively more impaired group than self-referred SCD cases.

While recruitment source did not significantly bias cognitive performance or demographics, we observed a relative increase in depressive symptoms measured by the GDS 15 in the memory clinic-referred SCD cases compared to self-referrals (data not shown). However, the observed increase in symptoms was not above the suggested cut-offs for clinical depression at group level [40]. This is not a surprising finding since severe psychiatric illness, including major depression, is a core exclusion criterion in this study. However, this may not be the case in all study designs investigating SCD cases. As such, recruitment from memory clinics may lead to inclusion of a higher percentage of clinically depressed individuals. The role of depressive symptoms in SCD and preclinical AD is however unclear [12]. A recent study by Perrotin et al. [20] comparing SCD cases recruited from memory clinics and community sample, showed significant reductions in gray matter volumes related to $\mathrm{AD}$ pathology in the memory clinic group. The authors conclude that medical help seeking and increased depressive symptoms were related to these volume reductions and pointing out increased affective burden as a potential part of prodromal AD. Conversely, Heser et al. [41] found that depressive symptoms were fully mediated by subjective memory impairment worry, suggesting that depressive symptoms were caused by an increased awareness of subjective decline, explaining levels of depressive symptoms in individuals with subjective cognitive complaints. As such, depressive symptoms even at subclinical levels may be an important factor in preclinical AD. This should be further explored in future studies focusing on the trajectory of preclinical AD development.
While not demonstrating statistically significant recruitment bias in cognitive performance, the present study shows that the SCD group performed worse on key cognitive domains associated with $\mathrm{AD}$ such as learning and executive functions, as well as a general decline in overall cognitive screening performance (MMSE) compared to controls. Although observed effect sizes were small, these findings support the notion that SCD could be a symptom of awareness of subtle cognitive decline witnessed by small declines in cognitive performance, while still performing within limits of normal variations [6]. A recent review by Garcia-Ptacek et al. [42] summarizes that, although most studies show poorer cognitive performance in persons with subjective cognitive complaints, such findings have not been universally supported. Furthermore, reductions within these domains may be influenced by other factors such as personality, anxiety or depressive symptoms, or accounted for by other medical or neurological disorders other than AD. Both CERAD word list learning and Trail making test $\mathrm{B}$ rely on adequate working memory and attentional processes, both of which can be affected by numerous conditions. Also, the SCD group did not perform worse than controls on memory recall. These findings are therefore not unequivocally linked to AD-pathology and may represent different conditions or disease etiologies. Moreover, the control and SCD group performed very similar in most of the cognitive measures, which may suggest that AD enrichment within the SCD group is relatively low. Future studies combining biological markers with SCD phenotypes and follow-ups are needed to ascertain the meaning of this finding.

The present study has some limitations that need to be addressed. First, due to geographic differences in Norway, the availability of memory clinics may differ. This could lead to a biased inclusion of memory clinic-referrals living in, or near city centers where the university hospitals are located. Second, our study is limited to a cross sectional comparison of cohort characteristics and does not include outcome measure of disease progression. Third, we did not include the use of biomarker evidence to further characterize selection bias, limiting interpretation of current findings. Fourth, the inclusion criteria allow the recruitment of younger middle aged adults (40-80 y), which lowers the mean age and increases variability in our sample. Advancing age is a well-known risk factor for AD. Thus, while this is an optimal design to capture early preclinical disease events in a longitudinal study, it may lead to dilution of AD prevalence in 
both SCD and MCI samples in our cross-sectional analysis. Lastly, an important note has to be made on the use of Sotaniemi et al. [34] CERAD word list normative dataset. These norms are based on a sample that is on average 10 years older and less educated than the DDI cohort. This may in some cases lead to uncertain classification of MCI and SCD. As such, there may be a need to establish normative datasets better suited for younger pre-clinical at-risk cohorts.

A central aim of the DDI project is to examine incipient disease activity to detect and track dementia disease progression in its preclinical states. Therefore, the cohort is comprised of a younger sample than most previous large cohort studies and the characteristics of this sample with regard to the impact of biomarker findings and longitudinal outcomes are not yet known. Future studies on the DDI cohort employing longitudinal designs and utilizing both biological and psychological data will serve to further delineate the clinical significance of these findings to define the characteristics of SCD due to AD more closely.

\section{Conclusions}

Our findings indicate that recruitment through memory clinics preferentially includes participants at higher risk of dementia, or are more advanced than cases recruited through other means. In addition, SCD cases were shown to perform worse on key cognitive measures compared to controls and may suggest that SCD is a symptom of subtle cognitive decline. Recruitment was not shown to significantly bias demographic characteristics or cognitive performance within the SCD group alone. However, at symptom group level, we did find an overall significant effect of worse cognitive performance in memory clinic-referrals beyond what was shown by the MCI group alone. This suggests that although the differences in cognitive performance are small, SCD cases recruited from memory clinics may represent a cognitively more impaired group than self-referred SCD cases. These findings suggest that recruitment source affects clinical characteristics of preclinical cohorts and should be taken into consideration when comparing findings between studies utilizing different recruitment methods. Future studies employing longitudinal designs and combining psychological and biological data are needed to further delineate the significance of these findings, as well as addressing the impact of recruitment bias on biological risk-factors within the DDI cohort.

\section{ACKNOWLEDGMENTS}

The project was funded by Norwegian Research Council, NASATS (Dementia Disease Initiation) and the JPND (APGeM) and funding from the regional health authorities (Helse Sør-Øst and Helse Nord). We thank Svein Ivar Bekkelund, Kjell-Arne Arntzen, Kai Müller, Torgil Riise Vangberg, Claus Albretsen, Elisabeth Gundersen, Mari Thoresen Løkholm, Ida Harviken, Line Sæther, Erna Utnes, Marianne Wettergreen, Berglind Gisladottir, Marit Knapstad, Reidun Meling, and Synnøve Bremer Skarpenes for clinical examinations and essential help with the project.

Authors' disclosures available online (http://j-alz. com/manuscript-disclosures/17-0385r1).

\section{REFERENCES}

[1] Petersen RC (2016) Mild cognitive impairment. Continuum (Minneap Minn) 22, 404-418.

[2] Albert MS, DeKosky ST, Dickson D, Dubois B, Feldman HH, Fox NC, Gamst A, Holtzman DM, Jagust WJ, Petersen RC, Snyder PJ, Carrillo MC, Thies B, Phelps CH (2011) The diagnosis of mild cognitive impairment due to Alzheimer's disease: Recommendations from the National Institute on Aging-Alzheimer's Association workgroups on diagnostic guidelines for Alzheimer's disease. Alzheimers Dement 7, 270-279.

[3] Perrin RJ, Fagan AM, Holtzman DM (2009) Multimodal techniques for diagnosis and prognosis of Alzheimer's disease. Nature 461, 916-922.

[4] Sperling RA, Aisen PS, Beckett LA, Bennett DA, Craft S, Fagan AM, Iwatsubo T, Jack CR Jr, Kaye J, Montine TJ, Park DC, Reiman EM, Rowe CC, Siemers E, Stern Y, Yaffe K, Carrillo MC, Thies B, Morrison-Bogorad M, Wagster MV, Phelps CH (2011) Toward defining the preclinical stages of Alzheimer's disease: Recommendations from the National Institute on Aging-Alzheimer's Association workgroups on diagnostic guidelines for Alzheimer's disease. Alzheimers Dement 7, 280-292.

[5] Sperling RA, Jack CR Jr, Aisen PS (2011) Testing the right target and right drug at the right stage. Sci Transl Med 3, $111 \mathrm{~cm} 33$.

[6] Jessen F, Amariglio RE, van Boxtel M, Breteler M, Ceccaldi M, Chételat G, Dubois B, Dufouil C, Ellis KA, van der Flier WM, Glodzik L, van Harten AC, de Leon MJ, McHugh P, Mielke MM, Molinuevo JL, Mosconi L, Osorio RS, Perrotin A, Petersen RC, Rabin LA, Rami L, Reisberg B, Rentz DM, Sachdev PS, de la Sayette V, Saykin AJ, Scheltens P, Shulman MB, Slavin MJ, Sperling RA, Stewart R, Uspenskaya O, Vellas B, Visser PJ, Wagner M, Subjective Cognitive Decline Initiative (SCID-I) Working Group (2014) A conceptual framework for research on subjective cognitive decline in preclinical Alzheimer's disease. Alzheimers Dement 10, 844-852.

[7] Reiman EM, Langbaum JB, Tariot PN, Lopera F, Bateman RJ, Morris JC, Sperling RA, Aisen PS, Roses AD, Welsh-Bohmer KA, Carrillo MC, Weninger S (2016) CAPadvancing the evaluation of preclinical Alzheimer disease treatments. Nat Rev Neurol 12, 56-61. 
[8] Karran E, De Strooper B (2016) The amyloid cascade hypothesis: Are we poised for success or failure? $\mathrm{J} \mathrm{Neu}$ rochem 139, 237-252.

[9] Visser PJ, Verhey F, Knol DL, Scheltens P, Wahlund LO, Freund-Levi Y, Tsolaki M, Minthon L, Wallin AK, Hampel H, Bürger K, Pirttila T, Soininen H, Rikkert MO, Verbeek MM, Spiru L, Blennow K (2009) Prevalence and prognostic value of CSF markers of Alzheimer's disease pathology in patients with subjective cognitive impairment or mild cognitive impairment in the DESCRIPA study: A prospective cohort study. Lancet Neurol 8, 619-627.

[10] van Harten AC, Visser PJ, Pijnenburg YA, Teunissen CE, Blankenstein MA, Scheltens P, van der Flier WM (2013) Cerebrospinal fluid $\mathrm{A} \beta 42$ is the best predictor of clinical progression in patients with subjective complaints. Alzheimers Dement 9, 481-487.

[11] Rönnlund M, Sundström A, Adolfsson R, Nilsson LG (2015) Subjective memory impairment in older adults predicts future dementia independent of baseline memory performance: Evidence from the Betula prospective cohort study. Alzheimers Dement 11, 1385-1392.

[12] Mendonça MD, Alves L, Bugalho P (2016) From subjective cognitive complaints to dementia: Who is at risk? A systematic review. Am J Alzheimers Dis Other Demen 31, 105-114.

[13] Bassett SS, Folstein MF (1993) Memory complaint, memory performance, and psychiatric diagnosis: A community study. J Geriatr Psychiatry Neurol 6, 105-111.

[14] Whitwell JL, Wiste HJ, Weigand SD, Rocca WA, Knopman DS, Roberts RO, Boeve BF, Petersen RC, Jack CR Jr, Alzheimer Disease Neuroimaging Initiative (2012) Comparison of imaging biomarkers in the Alzheimer Disease Neuroimaging Initiative and the Mayo Clinic Study of Aging. Arch Neurol 69, 614-622.

[15] Brodaty H, Mothakunnel A, de Vel-Palumbo M, Ames D, Ellis KA, Reppermund S, Kochan NA, Savage G, Trollor JN, Crawford J, Sachdev PS (2014) Influence of population versus convenience sampling on sample characteristics in studies of cognitive aging. Ann Epidemiol 24, 63-71.

[16] Farias ST, Mungas D, Reed BR, Harvey D, DeCarli C (2009) Progression of mild cognitive impairment to dementia in clinic- vs community-based cohorts. Arch Neurol 66, 1151-1157.

[17] Roh HW, Hong CH, Lee Y, Lee KS, Chang KJ, Kang DR, Lee JD, Choi SH, Kim SY, Na DL, Seo SW, Kim DK, Back JH, Chung YK, Lim KY, Noh JS, Son SJ (2016) Clinical conversion or reversion of mild cognitive impairment in community versus hospital based studies: GDEMCIS (Gwangju Dementia and Mild Cognitive Impairment Study) and CREDOS (Clinical Research Center for Dementia of South Korea). J Alzheimers Dis 53, 463-473.

[18] Rodriguez-Gómez O, Abdelnour C, Jessen F, Valero S, Boada M (2015) Influence of sampling and recruitment methods in studies of subjective cognitive decline. J Alzheimers Dis 48, S99-S107.

[19] Chen Y, Denny KG, Harvey D, Farias ST, Mungas D, DeCarli C, Beckett L (2017) Progression from normal cognition to mild cognitive impairment in a diverse clinic-based and community-based elderly cohort. Alzheimers Dement 13, 399-405.

[20] Perrotin A, La Joie R, de La Sayette V, Barré L, Mézenge F, Mutlu J, Guilloteau D, Egret S, Eustache F, Chételat G (2017) Subjective cognitive decline in cognitively normal elders from the community or from a memory clinic:
Differential affective and imaging correlates. Alzheimers Dement 13, 550-560.

[21] Abdelnour C, Rodriguez-Gómez O, Alegret M, Valero S, Moreno-Grau S, Sanabria Á, Hernández I, RosendeRoca M, Vargas L, Mauleón A, Sánchez D, Espinosa A, Ortega G, Perez-Cordón A, Diego S, Gailhajanet A, Guitart M, Sotolongo-Grau Ó, Ruiz A, Tárraga L, Boada M (2017) Impact of recruitment methods in subjective cognitive decline. J Alzheimers Dis 57, 625-632.

[22] Hessen E, Eckerström M, Nordlund A, Selseth Almdahl I, Stålhammar J, Bjerke M, Eckerström C, Göthlin M, Fladby T, Reinvang I, Wallin A (2017) Subjective cognitive impairment is a predominantly benign condition in memory clinic patients followed for 6 years: The Gothenburg-Oslo MCI Study. Dement Geriatr Cogn Dis Extra 7, 1-14.

[23] Fladby T, Pålhaugen L, Selnes P, Waterloo K, Bråthen G, Hessen E, Almdahl IS, Arntzen KA, Auning E, Eliassen CF, Espenes R, Grambaite R, Grøntved GR, Johansen KK, Johnsen SH, Kalheim LF, Kirsebom BE, Müller KI, Nakling EA, Rongve A, Sando SB, Siafarikas N, Stav AL, Tecelao S, Timon S, Bekkelund SI, Aarsland D (2017) Detecting at-risk Alzheimer's disease cases J Alzheimers Dis 60 , 97-105.

[24] Albert MS, DeKosky ST, Dickson D, Dubois B, Feldman HH, Fox NC, Gamst A, Holtzman DM, Jagust WJ, Petersen RC, Snyder PJ, Carrillo MC, Thies B, Phelps CH (2011) The diagnosis of mild cognitive impairment due to Alzheimer's disease: Recommendations from the National Institute on Aging-Alzheimer's Association workgroups on diagnostic guidelines for Alzheimer's disease. Alzheimers Dement 7, 270-279.

[25] Mitchell AJ, Bird V, Rizzo M, Meader N (2010) Diagnostic validity and added value of the Geriatric Depression Scale for depression in primary care: A meta-analysis of GDS30 and GDS15. J Affect Disord 125, 10-17.

[26] Heaton RK (2004) Revised Comprehensive Norms for an Expanded Halstead-Reitan Battery: Demographically Adjusted Neuropsychological Norms for African American and Caucasian Adults: Professional Manual, Psychological Assessment Resources.

[27] Folstein MF, Folstein SE, McHugh PR (1975) "Minimental state". A practical method for grading the cognitive state of patients for the clinician. J Psychiatr Res 12, 189-198.

[28] Shulman KI (2000) Clock-drawing: Is it the ideal cognitive screening test? Int J Geriatr Psychiatry 15, 548-561.

[29] Fillenbaum GG, van Belle G, Morris JC, Mohs RC, Mirra SS, Davis PC, Tariot PN, Silverman JM, Clark CM, WelshBohmer KA, Heyman A (2008) Consortium to Establish a Registry for Alzheimer's Disease (CERAD): The first twenty years. Alzheimers Dement 4, 96-109.

[30] Benton AL, Hamsher Kd (1989) Multilingual Aphasia Examination. AJA Associates, Iowa City.

[31] McKhann GM, Knopman DS, Chertkow H, Hyman BT, Jack CR Jr, Kawas CH, Klunk WE, Koroshetz WJ, Manly JJ, Mayeux R, Mohs RC, Morris JC, Rossor MN, Scheltens P, Carrillo MC, Thies B, Weintraub S, Phelps $\mathrm{CH}$ (2011) The diagnosis of dementia due to Alzheimer's disease: Recommendations from the National Institute on Aging-Alzheimer's Association workgroups on diagnostic guidelines for Alzheimer's disease. Alzheimers Dement 7, 263-269.

[32] Warrington EK, James M (1991) The Visual Object and Space Perception Battery, Thames Valley Test Company, Bury St Edmunds, England. 
[33] Reitan RM, Wolfson D (1985) The Halstead-Reitan Neuropsychological Test Battery, Neuropsychology Press, Tucson.

[34] Sotaniemi M, Pulliainen V, Hokkanen L, Pirttilä T, Hallikainen I, Soininen H, Hänninen T (2012) CERADneuropsychological battery in screening mild Alzheimer's disease. Acta Neurol Scand 125, 16-23.

[35] Hughes CP, Berg L, Danziger WL, Coben LA, Martin RL (1982) A new clinical scale for the staging of dementia. $\mathrm{Br}$ J Psychiatry 140, 566-572.

[36] Petersen RC (2004) Mild cognitive impairment as a diagnostic entity. J Intern Med 256, 183-194.

[37] Fritz CO, Morris PE, Richler JJ (2012) Effect size estimates: Current use, calculations, and interpretation. J Exp Psychol Gen 141, 2-18.

[38] Schneider JA, Aggarwal NT, Barnes L, Boyle P, Bennett DA (2009) The neuropathology of older persons with and without dementia from community versus clinic cohorts. J Alzheimers Dis 18, 691-701.
[39] Valenzuela MJ, Sachdev P (2006) Brain reserve and dementia: A systematic review. Psychol Med 36, 441-454.

[40] Marc LG, Raue PJ, Bruce ML (2008) Screening performance of the 15-Item Geriatric Depression Scale in a diverse elderly home care population. Am J Geriatr Psychiatry 16, 914-921.

[41] Heser K, Tebarth F, Wiese B, Eisele M, Bickel H, Köhler M, Mösch E, Weyerer S, Werle J, König HH, Leicht H, Pentzek M, Fuchs A, Riedel-Heller SG, Luppa M, Prokein J, Scherer M, Maier W, Wagner M, AgeCoDe Study Group (2013) Age of major depression onset, depressive symptoms, and risk for subsequent dementia: Results of the German study on Ageing, Cognition, and Dementia in Primary Care Patients (AgeCoDe). Psychol Med 43, 1597-1610.

[42] Garcia-Ptacek S, Eriksdotter M, Jelic V, Porta-Etessam J, Kåreholt I, Manzano Palomo S (2016) Subjective cognitive impairment: Towards early identification of Alzheimer disease. Neurología 31, 562-571. 